\title{
Past 3 Months
}

National Cancer Institute

\section{Source}

National Cancer Institute. Past 3 Months. NCI Thesaurus. Code C125104.

A period of time consisting of the 3 months before the present. 